\title{
The Prevalence and Prognostic Value of Neuroimaging Abnormalities in the Acute Phase of Sepsis-Associated Encephalopathy
}

\author{
Keenan A Walker ${ }^{1}$, Kaitlin Happer ${ }^{2}$, Rachel Venezia², Eric Gottesman ${ }^{3}$, Christopher G Filippi ${ }^{4}$ and Pina C Sanelli ${ }^{4}$ \\ ${ }^{1}$ Department of Neurology, Johns Hopkins School of Medicine, USA \\ ${ }^{2}$ Department of Psychology, St. John's University, USA \\ ${ }^{3}$ Department of Pulmonary Disease, Critical Care Medicine, Northwell Health, USA \\ ${ }^{4}$ Department of Radiology, Northwell Health, USA \\ *Corresponding author: Keenan Walker, Department of Neurology, Johns Hopkins Hospital, Phipps 446600 North Wolfe St., Baltimore, MD 21287, USA
}

Submission: September 23, 2017; Published: April 12, 2018

\begin{abstract}
Objectives: Sepsis-associated encephalopathy (SAE)is the most common form of encephalopathy in the intensive care unit (ICU) today. However, little is known about the underlying pathogenesis and clinical significance of the brain changes associated with SAE. Using magnetic resonance imaging (MRI) and computed tomography (CT), the present study evaluated the spectrum of brain abnormalities in patients with SAE and examined whether these abnormalities predicted clinical features of SAE, ICU mortality, one-year mortality, and disability.

Methods: We examined clinical and radiological data from 60 consecutive adults admitted to the medical ICU without preexisting neurological conditions, who met criteria severe sepsis or septic shock and subsequently developed SAE. Neuroimaging attributes were rated by two radiologists using a standardized protocol.

Results: Common neuroimaging findings included periventricular and subcortical white matter lesions, cerebrovascular calcification, and lacunar infarction. By comparison, acute infarction, acute hemorrhage, cytotoxic edema, and vasogenic edema were less prevalent. Acutely altered mental status (e.g., delirium) was the most common form of SAE and was associated with greatercerebral atrophy $(\mathrm{p}=.029)$ and subcortical white matter lesion burden $(\mathrm{p}=.010)$ relative to patients presenting with new-onset of focal neurologic signs or new-onset seizure. Acute, but not chronic, neuroimaging findings were associated with risk for ICU mortality ( $\mathrm{p}=.026)$, one-year mortality $(\mathrm{p}=.016)$, and disability $(\mathrm{p}=.032)$ after adjusting for potentially confounding variables.
\end{abstract}

Discussion: Though less common than chronic neuroimaging findings, acute neuroimaging abnormalities have prognostic significance as predictors of disability and survival in the first year following the development of SAE.

Keywords: Brain Disease; Brain Injuries, Acute; Neuroimaging; Critical Care; Sepsis; Shock, Septic

\section{Introduction}

Severe sepsis is a common form of critical illness that affects approximately 750,000 people in the USA annually [1]. Morbidity and mortality, especially within the first year after severe sepsis are exceedingly high, making sepsis the $10^{\text {th }}$ leading cause of death in the USA [1,2]. Acute cognitive or neurologic dysfunction during sepsis, known collectively as sepsis-associated encephalopathy (SAE), is the most common form of encephalopathy in the intensive care unit (ICU) [3-5]. Although it remains unclear whether the pathological processes associated with neurologic and cognitive dysfunction during sepsis are fully reversible, SAE has been linked to a two-fold increase in mortality and elevated risk for long-term cognitive deficits $[4,6,7]$. Currently, the ability to successfully treat and identify patients most vulnerable to SAE is limited by an incomplete knowledge of the underlying pathogenesis and neurobiology of this syndrome.

The current understanding of SAE is based largely on animal models and a set of descriptive case series [8-14]. To date, human studies of SAE have been limited by small sample size and the inclusion of heterogeneous patient groups with a range of medical and neurologic comorbidity. The only large neuroimaging study to examine brain changes in SAE found either leukoencephalopathy or ischemic stroke in approximately half of patients [5]. However, because this study did not make the distinction between acute and chronic neuroimaging abnormalities, the significance of 
these findings remains unclear. To date, the prevalence of other clinically relevant neuroimaging abnormalities, such as cytotoxic and vasogenicedema, has not been examined in a large series of patients with SAE, significantly limiting the understanding of SAE pathogenesis. The link between SAE and mortality among septic patients also remains poorly understood. Although ischemic stroke has been associated with increased ICU mortality [5], it is currently unknown whether specific neuroimaging abnormalities observed during the acute phase of SAE are predictive of survival within the first year following ICU discharge, a period when mortality is as high as $50 \%$ for individuals who survive sepsis [2].

The goals of the current study were to extend the understanding of SAE by using MRI and CT to evaluate the prevalence and clinical significance of acute and chronic brain abnormalities in patients who developed cognitive or neurological dysfunction (i.e., altered mental status, focal neurologic sign, or seizure) during the acute phase of severe sepsis or septic shock. To this end, we first examined the prevalence of vasogenic and cytotoxic edema, hemorrhage, infarction, atrophy, arterial and venous calcification, and white matter lesions. Based on previous findings [15-17], white matter lesions, acute infarctions, and cerebral edema were expected to be most common. The second goal of this study was to examine the association between clinical features of SAE and neuroimaging attributes with the expectation that focal neurologic findings and acutely altered mental status would be associated with acute neuroimaging findings and elevated white matter lesion burden, respectively. Lastly, we tested the hypothesis that acute neuroimaging findings and elevated white matter lesion burden would be associated with greater risk of ICU mortality, one-year mortality, and disability.

\section{Materials and Methods}

\section{Study Design and Population}

This was a retrospective analysis of data collected from consecutive patients admitted to a 17-bed ICU at an academic medical institution in the greater New York metropolitan area between January 2012 and June 2015. The study protocol was reviewed and approved by the hospital's institutional review board; HIPPA compliance was maintained. Relevant medical, demographic, and treatment-related data were extracted from electronic medical records. Mortality data were obtained from health system records and the Social Security Death Index.

Eligible patients met the following criteria:

a) Diagnosis of severe sepsis or septic shock according to International Sepsis Definitions Conference of 2001guidelines $[18,19]$;

b) Experienced acute cognitive or neurological dysfunction during the course of septicemia while in the ICU;

c) Received care in the ICU for longer than 72 hours;

d) Received at least one head CT or brain MRI during ICU stay following the development of SAE; and e) Between ages 18and 79 (inclusive).

The decision to perform brain imaging was based on the judgment of clinicians caring for the patient.

Patients with current or previous medical conditions (e.g., cardiac arrest) or treatments (e.g., chemotherapy) known to affect the structure or function central nervous system were excluded. Individuals 80 years of age or older were excluded due to an elevated risk of pre-ICU dementia or cerebrovascular disease. Severe sepsis was defined as sepsis associated with organ dysfunction. Septic shock was defined as the presences of sepsis with refractory arterial hypotension necessitating catecholamine infusion [7].

\section{Sepsis-Associated Encephalopathy}

SAE was defined as the development of one or more of the following conditions demonstrating acute central nervous system dysfunction concurrently with the onset of infection or during the course of sepsis:

A. Acutely altered mental status,

B. New-onset focal neurologic finding(s), or

C. New-onset seizure $[4,20,21]$.

Acutely altered mental status was defined as the development of coma, delirium, prolonged disorientation, misinterpretation of stimuli, hallucinations, delusions, or a change in sensorium [4]. Focal neurologic deficit was defined as any acute development of abnormalities on a neurological exam suggestive of brain, nerve, or spinal cord dysfunction. Seizure was defined as the transient occurrence of abnormal generalized tonic-clonic or recurrent focal motor or sensory abnormalities as observed and documented by a physician or nurse [22].

Each of these conditions were categorized as SAE if they were

1. Not previously documented in the patient's history prior to the ICU admission, and

2. Judged to have developed concomitantly with or following the onset of sepsis. Neurological exams, which consisted of an assessment of level of consciousness and arousal, orientation, speech, and ability to follow motor commands, were conducted approximately every four hours for all patients per standardof-care. The Confusion Assessment Method for the Intensive Care Unit (CAM-ICU) (28) was used to assess delirium in 60\% of patients. A validated chart-based method for identification of delirium [24] was used for patients without available CAM-ICU data.

\section{Physiological Data}

The Acute Physiology and Chronic Health Evaluation III (APACHE-III) [25] and Sequential Organ Failure Assessment (SOFA) [26] rating scales were used to quantify severity of systemic illness and organ dysfunction, respectively. The Charlson Comorbidity Index (CCI) was used to quantify the level of medical comorbidity [27]. A cardiovascular health score was generated by summing the weighted score of a subset of the CCI indices (i.e., myocardial 
infarction, congestive heart failure, peripheral vascular disease or bypass, hypertension, use of coagulation therapy, and diabetes).

\section{Neuroimaging}

CT Imaging Protocol. Non-contrast CT (NCCT) of the head was performed on a 64-slice multi detector scanner (General Electric Medical Systems, Milwaukee, WI) according to routine departmental protocol utilizing contiguous $5.0 \mathrm{~mm}$ axial sections from the foramen magnum to the vertex without the intravenous administration of contrast. Standardized scanner parameters were set at $120 \mathrm{kVp}, 200 \mathrm{~mA}$, and $1 \mathrm{sec}$ rotation time. MR Imaging Protocol. Non-contrast MR imaging of the head was performed on a 1.5T or 3.0T scanner (General Electric Medical Systems) according to routine scanning protocol utilizing sagittal T1-weighted image (WI), axial T1W, T2W, FLAIR, susceptibility and/or gradient echo, and diffusion-weighted imaging.

Two board certified radiologists with Certificates of Added Qualification (CAQ) in neuroradiology and over 15 years of experience reviewed all neuroimaging using a standardized rating form. Independent assessments of the CT or MR imaging examinations were performed blinded to the patient's clinical, treatment, and outcomes data. For patients who underwent multiple neuroimaging studies, only the first MRI and/or CT scans following the SAE event were rated. Nineteen scans were rated by both radiologists. The degree of agreement between raters ranged from "good" to "excellent" across all neuroimaging attributes (kappa scores: .68 - .89).

Patient MRI and CT scans were recorded as 'normal' and 'abnormal.' Brain atrophy was qualitatively graded as absent, mild, moderate, and severe. MRI and CT scans were also examined for the presence of lacunar infarction, petechial hemorrhage, gross hemorrhage, encephalomalacia, arterial/venous calcification, and cerebral edema. All edema was categorized as either cytotoxic or vasogenic in origin and categorized as generalized versus focal. The neuroanatomical location and number of each neuroimaging abnormality were recorded. Periventricular and deep subcortical white matter lesion burden was rated as absent, mild, moderate, and severe using the Fazekas scoring method [28], a visual rating system designed for use with MRI and CT scans. Cerebral infarction, gross hemorrhage, petechial hemorrhage, and cerebral edema were classified as either acute or non-acute.

\section{Statistical analysis}

Analyses were performed using Stata SE Version 14 (StataCorp, College Station, Texas).Comparisons of patient groups on demographic, medical, and treatment-related variables were conducted using chi-squared/Fisher's exact test and Mann-Whitney $\mathrm{U}$ tests for categorical and continuous variables, respectively. Associations between SAE characteristics and neuroimaging attributes were evaluated using one-way between-group ANOVAs and chi-square tests. Multivariable adjusted logistic regression and Cox regression analyses were used to assess the association between acute and chronic neuroimaging findings, and ICU mortality, hospital discharge disposition, and one-year mortality. Covariates for logistic and Cox regression analyses included: age, sex, disease severity (day 1 APACHE-III), and cardiovascular disease (cardiovascular health score). All analyses were initially conducted using aggregated data from MRI and CT scans. To account for potential differences related to imaging modality, planned sensitivity analyses were also conducted using only data from CT imaging. Because of limited sample size, sensitivity analyses using only MRI data were not performed. All tests were two-sided, and $\mathrm{p}$ $<.05$ was considered statistically significant.

\section{Results}

\section{Patient demographic and clinical data}

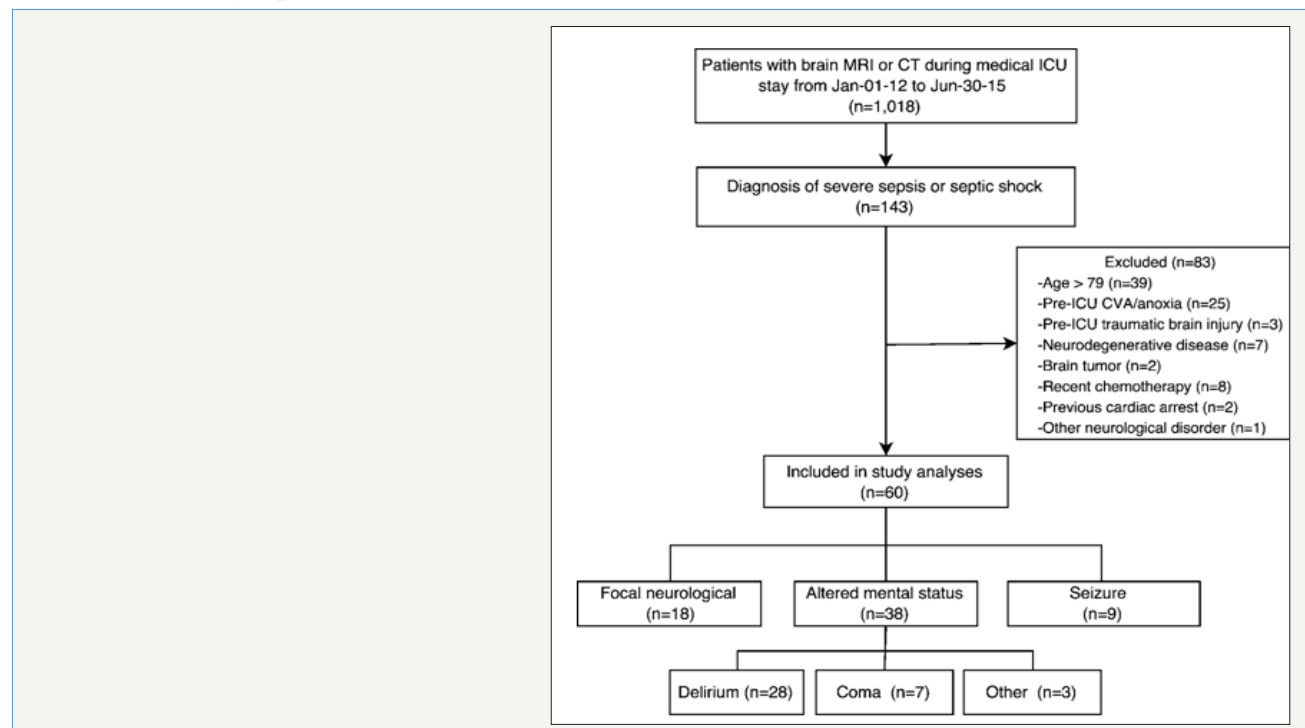

CT: Computed Tomography; CVA: Cerebrovascular Accident; ICU: Intensive Care Unit; MRI: Magnetic Resonance Imaging

Figure 1: Study enrollment flowchart. 
Table 1: Sample demographic, medical, and treatment-related characteristics stratified according to the presence of acute neuroimaging findings and level of white matter lesion burden.

\begin{tabular}{|c|c|c|c|c|c|c|}
\hline Characteristic & $\begin{array}{l}\text { Acute Imaging } \\
\text { Findings } \\
(n=13)\end{array}$ & $\begin{array}{l}\text { No Acute Imaging } \\
\text { Findings } \\
(n=47)\end{array}$ & $\mathbf{p}$ & $\begin{array}{c}\text { Moderate or } \\
\text { Severe WM } \\
\text { Lesions } \\
(n=19)\end{array}$ & $\begin{array}{l}\text { Absent or Mild } \\
\text { WM Lesions } \\
\text { (n= 41) }\end{array}$ & $\mathbf{p}$ \\
\hline Age - years & $66(52-75)$ & $67(62-74)$ & .82 & $73(67-76)$ & 65 (54-72) & .004 \\
\hline $\begin{array}{c}\text { Caucasian race }-\mathrm{n} \\
(\%)\end{array}$ & $8(62)$ & $23(49)$ & .99 & $8(67)$ & $23(74)$ & .62 \\
\hline Male sex - n (\%) & $8(62)$ & $29(62)$ & .96 & $15(79)$ & $22(54)$ & .06 \\
\hline $\begin{array}{l}\text { ICU Length of Stay } \\
\text { - days }\end{array}$ & $6(5-15)$ & $9(5-17)$ & .58 & $8(4-18)$ & $9(5-17)$ & .54 \\
\hline $\begin{array}{l}\text { Hospital Length of } \\
\text { Stay - days }\end{array}$ & $12(7-36)$ & $22(11-46)$ & .16 & $15(9-33)$ & $23(9-47)$ & .38 \\
\hline $\begin{array}{l}\text { ICU Days Before } \\
\text { CT/MRI }\end{array}$ & $4(1-6)$ & $3(1-9)$ & .44 & $4(1-9)$ & $3(1-9)$ & .62 \\
\hline $\begin{array}{l}\text { APACHE III score, } \\
\text { ICU day } 1 \\
\end{array}$ & $90(72-110)$ & $90(90-108)$ & .94 & $85(72-93)$ & $91(72-116)$ & .37 \\
\hline $\begin{array}{l}\text { SOFA, ICU day } 1 \text { ( } 0 \\
\text { to } 24 \text { ) }\end{array}$ & $8(6-11)$ & $8(6-11)$ & .96 & $7(5-10)$ & $8(6-11)$ & .58 \\
\hline $\begin{array}{l}\text { SOFA, ICU day } 3 \text { (0 } \\
\text { to } 24 \text { ) }\end{array}$ & $9(5-9)$ & $7(4-11)$ & .74 & $7(4-10)$ & $7(5-11)$ & .77 \\
\hline $\begin{array}{c}\text { Charlson } \\
\text { Comorbidity Index } \\
\end{array}$ & $4(3-6)$ & $4(4-8)$ & .42 & $5(4-8)$ & $5(4-7)$ & .73 \\
\hline \multicolumn{7}{|l|}{$\begin{array}{c}\text { Critical Illness - n } \\
(\%)\end{array}$} \\
\hline Severe sepsis & $2(15)$ & $12(26)$ & .52 & $5(26)$ & $8(20)$ & .55 \\
\hline Septic shock & $11(85)$ & $35(75)$ & .52 & $14(74)$ & $33(80)$ & .55 \\
\hline Cardiac & $3(23)$ & 7 (15) & .48 & $4(21)$ & $6(15)$ & .54 \\
\hline $\begin{array}{c}\text { Respiratory } \\
\text { distress / failure }\end{array}$ & $8(62)$ & $26(55)$ & .69 & $11(58)$ & $23(56)$ & .90 \\
\hline Acute kidney injury & $4(31)$ & $20(42)$ & .44 & $6(32)$ & $18(44)$ & .37 \\
\hline Acute liver failure & $1(8)$ & $2(4)$ & .62 & $0(0)$ & $3(7)$ & .23 \\
\hline $\begin{array}{l}\text { Multiple organ } \\
\text { dysfunction }\end{array}$ & $5(39)$ & $15(32)$ & .66 & $5(26)$ & $15(37)$ & .43 \\
\hline \multicolumn{7}{|l|}{$\begin{array}{c}\text { Co-morbid } \\
\text { Conditions - n (\%) }\end{array}$} \\
\hline Cardiac & $4(31)$ & $16(34)$ & .83 & $10(53)$ & $10(24)$ & .03 \\
\hline Pulmonary & $2(15)$ & $13(28)$ & .37 & $7(37)$ & $8(20)$ & .15 \\
\hline Kidney disease & $5(39)$ & $22(47)$ & .59 & $5(26)$ & $22(54)$ & .05 \\
\hline Liver disease & $1(8)$ & $5(11)$ & .75 & $1(5)$ & $5(12)$ & .41 \\
\hline Diabetes & $8(62)$ & $23(49)$ & .42 & $13(68)$ & $18(44)$ & .08 \\
\hline Cancer & $1(10)$ & $11(23)$ & .21 & $3(16)$ & $9(22)$ & .58 \\
\hline $\begin{array}{l}\text { Cardiovascular } \\
\text { health score }\end{array}$ & $2(2-4)$ & $2(2-4)$ & .97 & $3(2-5)$ & $2(1-3)$ & .023 \\
\hline \multicolumn{7}{|l|}{$\begin{array}{l}\text { Mechanical } \\
\text { ventilation }\end{array}$} \\
\hline $\begin{array}{c}\text { Number of patients } \\
(\%)\end{array}$ & $11(85)$ & $37(79)$ & .64 & $16(84)$ & $32(78)$ & .58 \\
\hline
\end{tabular}




\begin{tabular}{|c|c|c|c|c|c|c|}
\hline Number of days & $4(3-9)$ & $7(2-17)$ & .22 & $5(2-14)$ & $6(2-16)$ & .56 \\
\hline Delirium & $8(62)$ & $32(68)$ & .69 & $14(73)$ & $26(63)$ & .43 \\
\hline $\begin{array}{c}\text { Number of patients } \\
(\%)\end{array}$ & $2(2-7)$ & $3(3-5)$ & .99 & $3(1-5)$ & $2(0-5)$ & .73 \\
\hline $\begin{array}{c}\text { Number of days } \\
\text { Sedation days }\end{array}$ & $5(1-9)$ & $7(3-13)$ & .35 & $6(3-14)$ & $7(2-11)$ & .86 \\
\hline
\end{tabular}

APACHE: Acute Physiology and Chronic Health Evaluation - Third Edition; SOFA: Sequential Organ Failure Assessment

Notes: Descriptive data are provided in the form of median followed by (interquartile range; IQR) unless otherwise specified. Patients with Fazekas scores $>3$ were classified as having moderate to severe white matter lesion burden. $\square^{2} /$ Fisher's exact test and the MannWhitney $U$ were used to compare groups on categorical and continuous variables, respectively.

Between January 1, 2012, and June30, 2015, 60 patients were identified for inclusion (Figure 1). Patient demographic and clinical characteristics are presented in Table 1 . The prevalence of SAE clinical features is described in Figure 1. Eight percent $(5 / 60)$ of patients displayed more than one form of SAE before neuroimaging, and $27 \%(16 / 60)$ developed more than one form of SAE during the entirety of the ICU admission. The median number of days between ICU admission and identification of SAE was 2 for patients presenting with acutely altered mental status and seizure, and 3 for patients presenting with a focal neurologic sign. Patients were in the ICU for a median of 3 days before receiving neuroimaging. No adverse events were reported during the transport to or from the radiological suite or during imaging.

\section{Neuroimaging Findings}

Eighty-three percent $(50 / 60)$ of patients received one or more head CT;28\% (17/60) received one or more MRI; 12\% (7/60) received both a CT and an MRI. The prevalence rates of specific neuroimaging findings are displayed in Figure 2. Abnormal findings were present in $82 \%(49 / 60)$ of patients. Cerebral atrophy was present in 75\% (45/60) of patients. Among patients with cerebral atrophy, severity ranged from mild $(66 \%, 30 / 45)$ to moderate $(33 \%, 15 / 45)$. Subcortical lesions, periventricular lesions, and arterial and venous calcification were most common in the frontal lobe. Lacunar infarcts were most common in the basal ganglia.

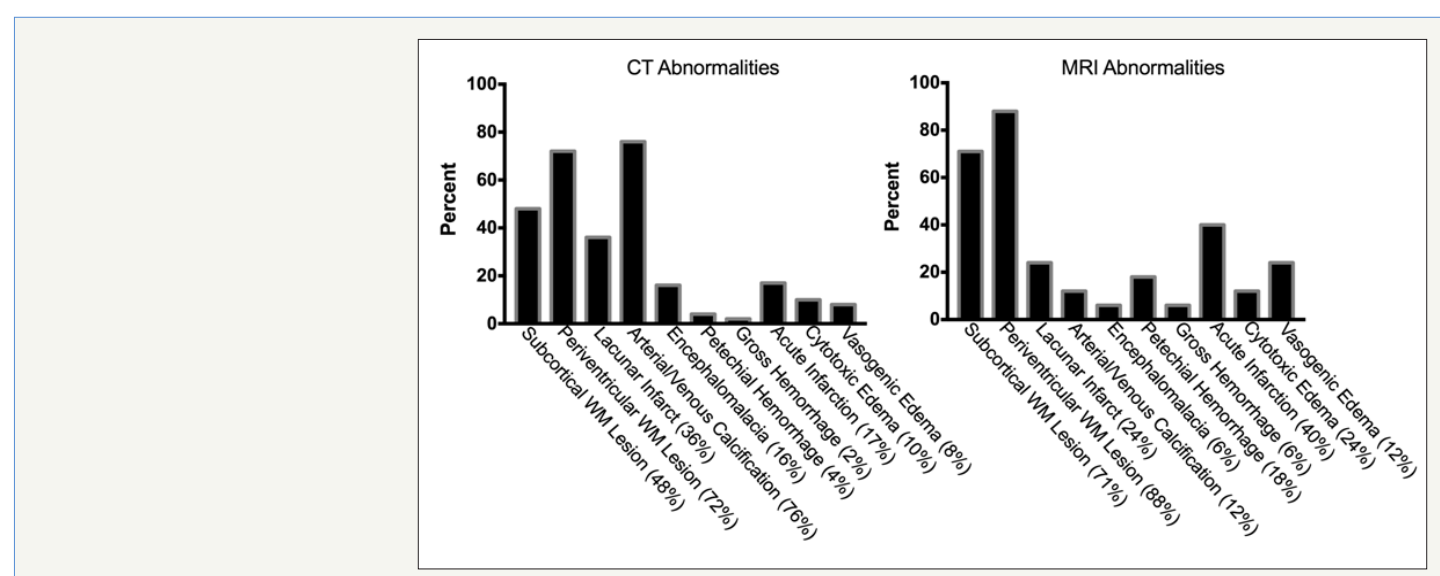

WM: White Matter

Figure 2: The prevalence of neuroimaging abnormalities among patients in the acute phase of sepsis-associated encephalopathy.

Acute neuroimaging findings were present in $22 \%(13 / 60)$ of patients. Among 11patients with acute infarction or hemorrhage, only three $(27 \%)$ demonstrated evidence of new-onset focal neurological findings. Acute infarctions were unilateral in the majority of cases $(73 \%, 8 / 11)$, and found most commonly in the frontal $(55 \%, 6 / 11)$ and parietal lobes $(36 \%, 4 / 11)$. Vasogenic edema was most common in the frontal $(100 \%, 6 / 6)$ and temporal lobes $(67 \%, 4 / 6)$. Cytotoxic edema was found at similar rates in the frontal $(50 \%, 4 / 8)$, occipital $(50 \%, 4 / 8)$, and parietal lobes(38\%, $3 / 8)$. Edema was typically focal (80\%-100\%) and strongly associated with the presence of an acute infarction or hemorrhage $\left(\chi^{2}=13.66, p<.001\right)$. Patients with acute neuroimaging findings did not differ from those without acute findings on demographic, medical, and treatment-related variables (Table 1).

Seventy-seven percent (46/60) of patients demonstrated white matter lesions on neuroimaging. Compared to patients with mild or absent white matter lesion burden (Fazekas score $\leq 3$ ), patients with moderate to severe white matter lesion burden were older (Mann-Whitney U = 209.00, p = .004), more likely to have comorbid cardiac disease $\left(\chi^{2}=4.66, p<.03\right)$, and had a greater cardiovascular risk profile (Mann-Whitney $U=249.50, p=.023$ ) (Table 1).

When patients were categorized according to the presenting clinical features of SAE and compared on neuroimaging attributes, 
patient groups differed in regards to the level of cerebral atrophy $(\mathrm{F}[2,57]=3.78, p=.029)$ and subcortical white matter lesion burden $(\mathrm{F}[2,57]=4.95, p=.010)$, but did not differ on other neuroimaging attributes. Follow-up univariate analyses revealed that patients who presented with acutely altered mental status had greater levels of cortical atrophy and greater subcortical white matter lesion burden relative to patients who presented with focal neurologic deficits or seizures $\left(p^{\prime} s<.05\right)$.

\section{Neuroimaging predictors of patient outcome}

ICU mortality for the current sample was 17\% (10/60), and one-year mortality was $40 \%$ (24/60). In a logistic regression model that adjusted for potential confounders (i.e., age, sex, APACHE-3 disease severity, and cardiovascular disease), the presence of an acute neuroimaging abnormality (i.e., infarction, hemorrhage, or edema) was associated with increased risk for ICU mortality (OR, 5.82, 95\% CI, 1.23 to 27.56, $p=.026$ ).In a covariate-adjusted Cox regression model, the presence of an acute neuroimaging abnormality was also associated with increased risk for one-year mortality following ICU discharge (HR, 3.09, 95\% CI, 1.23 to 7.72, $p=.016)$. Kaplan-Meier survival curves are displayed in Figure 3. By comparison, total white matter lesion burden was not associated with either ICU mortality or one-year mortality. The relationships between neuroimaging attributes, ICU mortality, and one-year mortality were unchanged in sensitivity analyses that included only data from CT imaging (Table 2).

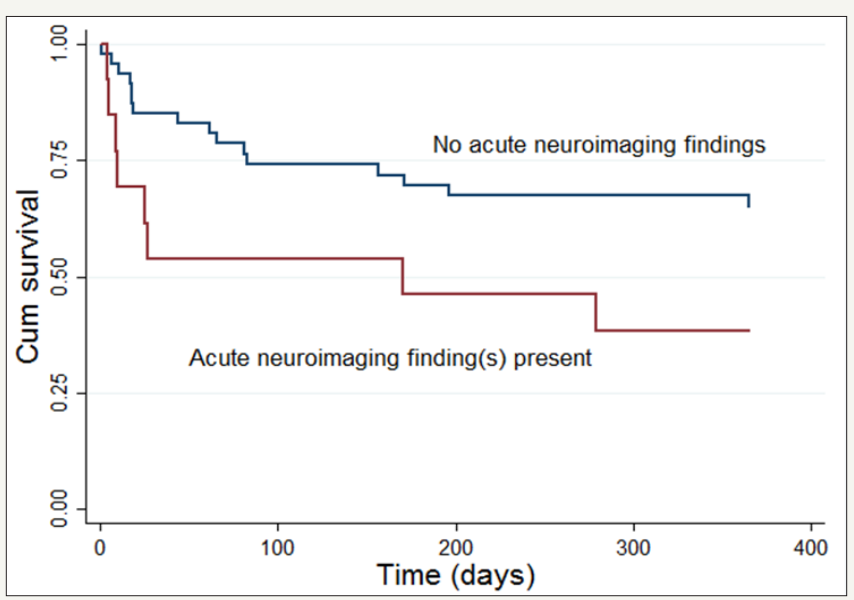

Figure 3: Kaplan-Meier survival curves evaluating the time to death in days for patients with and without acute neuroimaging abnormalities on MRI and/or CT scan following the onset of sepsis-associated encephalopathy ( $p=.042$ by log rank test).

Table 2: Associations between neuroimaging variables and ICU mortality, one-year mortality, and disability at hospital discharge

\begin{tabular}{|c|c|c|c|}
\hline Neuroimaging Variables & $\begin{array}{c}\text { ICU Mortality } \\
\text { OR (95\% CI) }\end{array}$ & $\begin{array}{c}\text { One-Year Mortality } \\
\text { HR (95\% CI) }\end{array}$ & $\begin{array}{c}\text { Disability } \\
\text { OR (95\% CI) }\end{array}$ \\
\hline $\begin{array}{c}\text { Acute Neuroimaging Abnormalities } \\
\text { (CT \& MRI) }\end{array}$ & $5.82(1.23-27.56)^{*}$ & $3.09(1.23-7.72)^{*}$ & $6.52(1.17-36.23)^{*}$ \\
\hline $\begin{array}{c}\text { Acute Neuroimaging Abnormalities } \\
\text { (CT only) }\end{array}$ & $7.28(1.26-41.85)^{*}$ & $4.28(1.51-12.14)^{* *}$ & $1.55(.69-62.39) t$ \\
\hline $\begin{array}{c}\text { Moderate to Severe White Matter } \\
\text { Lesions (CT \& MRI) }\end{array}$ & $2.67(.47-15.12)$ & $1.52(.59-4.46)$ & $.92-5.93)$ \\
\hline $\begin{array}{c}\text { Moderate to Severe White Matter } \\
\text { Lesions (CT only) }\end{array}$ & $1.58(.14-23.73)$ & $.19-4.21)$ \\
\hline
\end{tabular}

${ }^{\mathrm{t}} \mathrm{p}<.10 ;{ }^{*} \mathrm{p}<.05 ;{ }^{* *} \mathrm{p}<.01$

Notes: All models are adjusted for age, sex, cardiovascular disease, and day 1 disease severity (APACHE-III score). CI: Confidence Interval; HR: Hazard Ratio; OR: Odds Ratio

Of the patients discharged from the hospital, $64 \%(25 / 39)$ were discharged home (i.e., routine discharge) and 36\% (14/39) required continued comprehensive medical and rehabilitation services. In a covariate-adjusted logistic regression model, the presence of an acute neuroimaging abnormality was independently associated with an increased need for comprehensive medical and rehabilitation services following hospital discharge (OR, 6.52, 95\% CI, 1.17-36.23 p=.032). The association between acute neuroimaging findings and disposition at discharge did not change in a sensitivity analysis that included only data from CT imaging.
No association was found between white matter lesion burden and discharge disposition.

\section{Discussion}

In this study, we demonstrated that periventricular and subcortical white matter lesions, cerebral atrophy, and cerebrovascular calcification are the most common neuroimaging findings in patients with SAE, whereas acute neuroimaging findings, such as infarction and edema, are only found in a quarter of patients. Patients who developed acutely altered mental status 
were found to have more severe cortical atrophy and greater white matter lesion burden relative to patients who developed other forms of SAE. However, no specific neuroimaging finding was associated with the development of new-onset focal neurologic signs or seizure. Additionally, we demonstrated that the presence of acute, but not chronic, neuroimaging findings during the course of SAE is associated with increased risk for ICU mortality, greater disability at the time of hospital discharge, and reduced survival during the subsequent year.

The only previous study to examine neuroimaging abnormalities in a large series of patients with SAE found evidence of ischemic stroke in $29 \%$ of patients, approximately double that of the present study (15\%) [5]. Whereas the current study explicitly differentiated between acute infarctions and chronic lesions, it is unclear whether the ischemic strokes identified in this prior study represent acute infarctions, chronic silent infarctions, or both. Additionally, differences in stroke detection maybe due, in part, to differences in scan technique and/or imaging modality. The percentages of patients with severe periventricular (12\%) and subcortical (17\%) white matter abnormalities in the current study are similar to that which has been reported previously [5].

Given the age and prevalence of cardiovascular disease within our sample, it is likely that the white matter lesions and cerebral atrophy observed on neuroimaging predate the onset of sepsis [29]. However, in a previous study of ICU patients with acute neurologic changes (only $28 \%$ of whom were septic), the prevalence of white matter lesions and cerebral atrophy was $30 \%$ and $41 \%$, respectively [30], compared to $77 \%$ and $75 \%$, respectively, in the current study. Given these discrepancies, it is possible that the comparatively higher rates of white matter abnormalities and cerebral atrophy found in the current study are due to neuropathological processes unique to sepsis [8].Whereas fluid redistribution, steroid use, and hypoxic ischemia have each been proposed as potential causes of rapid cerebral atrophy, blood-brain barrier dysfunction, reduced cerebral perfusion, and neurotoxic inflammatory mediators have each been identified as possible causes of white lesions in septic patients [15,16,31-36]. On the other hand, it is also possible that chronic white matter lesions and cerebral atrophy may predispose septic patients to developing SAE.

The ability to simultaneously examine a large number of potentially relevant neuroimaging biomarkers represents a strength of the current study. Although cerebral edema has been previously examined in a heterogeneous group of ICU patients [30], the present study is the first to assess the prevalence of cytotoxic and vasogenicedema in a large series of patients with SAE. Animal research and number of human case studies suggest that cerebral edema may occur commonly in patients with sepsis and SAE $[9,37,38]$. Similarly, previous literature also suggests that sepsis is a risk factor for posterior reversible encephalopathy syndrome (PRES), a neurotoxic state characterized by significant vasogenic edema [39]. However, the current results indicate that cytotoxic and vasogenicedema may only appearin $10 \%$ to $24 \%$ of patients with SAE, depending on the neuroimaging modality. The present study is also the first to demonstrate high rates of cerebrovascular calcification among patients with SAE. Vascular calcification, a biological process marked by the deposition of calcium mineral deposits on intimal and medial layers of the vascular wall, is a common finding among middle-aged and older adults [40]. Vascular calcification can reduce arterial elasticity, contribute to atherosclerotic plaque burden, and in doing so, can promote inflammation and alter cerebral hemodynamics [41-43]. It is currently unclear whether cerebrovascular calcification increases one's risk for SAE.

The current results demonstrate that there is not necessarily a link between the clinical presentation of SAE and underlying neuroimaging abnormalities. Furthermore, our results suggest that neuroimaging abnormalities are not necessary for the development of SAE. However, the finding that only $33 \%$ of the patients with acute infarctions displayed focal neurologic signs underscores the importance of using neuroimaging for all forms of SAE [44]. Use of diffusion-weighted MRI (DWI) in patients with SAE may be especially helpful in the early identification of acute findings such as cytotoxic edema and acute infarction, which have prognostic significance as markers of disability and mortality [45]

Rates of mortality in the year following severe sepsis and septic shock are exceedingly high [2]. While it has been established that SAE is a risk factor for ICU mortality among patients with sepsis, the present study is the first to demonstrate that patients with SAE who develop acute neuroimaging abnormalities are at an elevated risk for mortality in the year following ICU discharge. In addition to highlighting the prognostic significance of acute neuroimaging findings among patients who develop SAE, the current results suggest that neurobiological processes related to SAE may directly influence survival of septic patients well after they leave the ICU. The exact nature of such a biological process remains unclear. However, mounting evidence suggests that cerebral infarction is associated with the depression of cell-mediated immune functioning [46,47], which may, in turn, increase susceptibility to infection $[48,49]$ and subsequent mortality.

\section{Limitations}

The current findings should be interpreted within the context of several limitations, including a retrospective study design, a relatively small sample size, and a lack of baseline pre-critical illness neuroimaging. Larger prospective studies will be needed to confirm and extend the present results. Additionally, because only patients with overt SAE that necessitated neuroimaging for clinical purposes (as judged by a treating physician) were included in the study, patients with more subtle forms of SAE may have been overlooked. Similarly, there may be a subset of patients who were either too medically unstable to undergo a CT or MRI or who died before necessary neuroimaging could be performed. In general, CT scans are less sensitive than MR imaging for the detection of brain abnormalities [45]. Thus, the current study may have underestimated the true prevalence of acute and chronic neuroimaging findings. However, with the exception of 
arterial and venous calcification, the prevalence of neuroimaging abnormalities did not differ significantly between patients with available MRI and patients with only CT imaging. Moreover, sensitivity analyses were used in the current study to confirm that CT imaging findings had similar prognostic significance to that of CT and MRI combined. The results of these sensitivity analyses are clinically relevant, as CT imaging is used more frequently than MRI to detect neurologic abnormalities in the acute medical ICU setting. Although neuroimaging is a powerful tool for the assessment of the pathological processes, many potential causes of SAE, including neurotransmitter abnormalities, microcirculatory and endothelial dysfunction, and changes in blood brain barrier permeability, are not directly observable using traditional structural imaging methods $[9,20,32,50]$. Future studies should make use of alternative imaging modalities capable of observing these neuropathological processes [16,51-53].

The multi-method approach to the assessment of delirium represents another limitation. Although the use of two distinct methods to assess delirium is not ideal, evidence suggests that the CAM-ICU and the chart-based delirium assessment method are highly comparable $[23,24]$. Lastly, more definitive conclusions about the pathophysiology of SAE could be reached if septic patients with and without SAE could be directly compared on the neuroimaging variables evaluated in the current study. However, given the life-threatening nature of critical illness, asking septic patients to undergo neuroimaging that is not clinically indicated may not be feasible.

\section{Conclusion}

Current findings suggest that SAE is a heterogeneous clinical syndrome that occurs early in the course of sepsis. Whereas chronic neuroimaging abnormalities such as cerebral atrophy, white matter lesions, and cerebrovascular calcification were found in the majority of patients, acute neuroimaging abnormalities, such as infarction, hemorrhage, and cerebral edema were less common. In general, neuroimaging findings do not necessarily correspond to specific clinical features of SAE. However, acute imaging finding, when present, are predictive of worse hospital outcomes and oneyear mortality, highlighting the importance of neuroimaging for purposes of prognostication and clinical decision making. Future prospective multisite studies that implement more sensitive neuroimaging techniques will be especially helpful in corroborating our findings and furthering the understanding of the most common form of encephalopathy in the ICU.

\section{Disclosure Statement}

No potential conflict of interest was reported by the authors.

\section{References}

1. Angus DC, Linde-Zwirble WT, Lidicker J, Clermont G, Carcillo J, et al (2001) Epidemiology of severe sepsis in the United States: analysis of incidence, outcome, and associated costs of care. Crit. Care Med 29(7): 1303-1310.

2. Puskarich M, Marchick M, Kline J, Steuerwald M, Jones A (2009) One year mortality of patients treated with an emergency department based early goal directed therapy protocol for severe sepsis and septic shock: a before and after study. Crit Care 13(5): R167.

3. Bleck TP, Smith MC, Pierre-Louis SJ, Jares JJ, Murray J, et al. (1993) Neurologic complications of critical medical illnesses. Crit Care Med 21(1): 98-103.

4. Sprung CL, Peduzzi PN, Shatney CH, Schein RM, Wilson MF, et al. (1990) Impact of encephalopathy on mortality in the sepsis syndrome. Crit Care Med 18(8): 801-806.

5. Polito A, Eischwald F, Maho A-L Le, Polito A, Azabou E, Annane D, et al. (2013) Pattern of Brain Injury in the Acute Setting of Human Septic Shock. Crit Care 17(5): R204.

6. Eidelman L a, Putterman D, Putterman C, Sprung CL (1996) The Spectrum of Septic Encephalopathy. JAMA 275(6): 470-473.

7. Pandharipande PP, Girard TD, Jackson JC, Morandi A, Thompson JL, et al. (2013) Long-term cognitive impairment after critical illness. N Engl J Med 369:1306-1316.

8. Jackson J, Hopkins R, Russell R, Gordon S, Wheeler A, Ely W (2009) Acute respiratory distress syndrome, sepsis, and cognitive decline: a review and case study. South Med 102(11): 1150-1157.

9. Piazza O, Cotena S, De Robertis E, Caranci F, Tufano R (2009) Sepsis associated encephalopathy studied by MRI and cerebral spinal fluid S100B measurement. Neurochem Res 34(7): 1289-1292.

10. Sharshar T, Gray F, Poron F, Raphael JC, Gajdos P, et al. (2002) Multifocal necrotizing leukoencephalopathy in septic shock. Crit Care Med 30(10): 2371-2375.

11. Rosengarten B, Hecht M, Auch D, Ghofrani HA, Schermuly RT, et al. (2007) Microcirculatory dysfunction in the brain precedes changes in evoked potentials in endotoxin-induced sepsis syndrome in rats. Cerebrovasc Dis 23(2-3): 140-147.

12. Morandi A, Gunther ML, Vasilevskis EE, Girard TD, Hopkins RO, et al. (2010) Neuroimaging in delirious intensive care unit patients: a preliminary case series report. Psychiatry (Edgmont) 7(9): 28-33.

13. Abe S, Okumura A, Fujii T, Someya T, Tadokoro R, et al. (2008) Sepsis associated encephalopathy in an infant with biliary atresia. Brain Dev 30(8): 544-547.

14. Kondo A, Sugiura C, Fujii Y, Inoue T, Maegaki Y, et al. (2009) Fulminant sepsis-associated encephalopathy in two children: Serial neuroimaging findings and clinical course. Neuropediatrics 40(4): 157-161.

15. Finelli PF, Uphoff DF (2004) Magnetic resonance imaging abnormalities with septic encephalopathy. J Neurol Neurosurg Psychiatry 75(8): 1189 1191.

16. Stubbs DJ, Yamamoto AK, Menon DK (2013) Imaging in sepsis-associated encephalopathy--insights and opportunities. Nat Rev Neurol 9(10): 551561.

17. Sharshar T, Carlier R, Bernard F, Guidoux C, Brouland JP, et al. (2007) Brain lesions in septic shock: A magnetic resonance imaging study. Intensive Care Med 33(5): 798-806.

18. Bone R, Robert B, Cerra F, Dellinger R, Fein A, Knaus W, et al. (1992) Definitions for sepsis and organ failure and guidelines for the use of innovative therapies in sepsis. The ACCP/ SCCM Consensus Conference Committee American College of Chest Physicians/Society of Critical Care Medicine. Chest 101(6): 1644-1655.

19. Levy MM, Fink MP, Marshall JC, Abraham E, Angus D, et al. (2003) 2001 SCCM/ESICM/ACCP/ATS/SIS International Sepsis Definitions Conference. Crit Care Med 31(4): 1250-1256.

20. Papadopoulos MC, Davies DC, Moss RF, Tighe D, Bennett ED (2000) Pathophysiology of septic encephalopathy: a review. Crit Care Med 28(8): 3019-3024. 
21. Lamar CD, Hurley RA, Taber KH (2011) Sepsis-associated encephalopathy: review of the neuropsychiatric manifestations and cognitive outcome. J Neuropsychiatry Clin Neurosci 23(3): 237-241.

22. Fisher RS, Acevedo C, Arzimanoglou A, Bogacz A, Cross H, et al. (2014) ILAE official report: a practical clinical definition of epilepsy. Epilepsia 55(4): 475-482.

23. Ely EW, Inouye SK, Bernard GR, Gordon S, Francis J, et al. (2001) Delirium in mechanically ventilated patients: Validity and reliability of the confusion assessment method for the intensive care unit (CAM-ICU). JAMA 286: 2703-2710.

24. Inouye SK, Leo-Summers L, Zhang Y, Bogardus ST, Leslie DL, et al (2005) A Chart-Based Method for Identification of Delirium: Validation Compared with Interviewer Ratings Using the Confusion Assessment Method. J Am Geriatr Soc Blackwell Science Inc 53(2): 312-318.

25. Knaus WA, Wagner DP, Draper EA, Zimmerman JE, Bergner M, et al. (1991) The APACHE III prognostic system. Risk prediction of hospital mortality for critically ill hospitalized adults. Chest 100: 1619-1636.

26. Ferreira FL, Bota DP, Bross A, Mélot C, Vincent JL (2001) Serial evaluation of the SOFA score to predict outcome in critically ill patients. JAMA 286(14): 1754-1758.

27. Charlson M, Szatrowski TP, Peterson J, Gold J (1994) Validation of a combined comorbidity index. J Clin Epidemiol 47(11): 1245-1251.

28. Wahlund LO, Barkhof F, Fazekas F, Bronge L, Augustin M, et al. (2001) A new rating scale for age-related white matter changes applicable to MRI and CT. Stroke 32(6): 1318-1322.

29. Vernooij MW, Ikram MA, Tanghe HL, Vincent AJPE, Hofman A, et al. (2007) Incidental findings on brain MRI in the general population. N Engl J Med 357: 1821-1828.

30. Suchyta MR, Jephson A, Hopkins RO (2010) Neurologic Changes during Critical Illness: Brain Imaging Findings and Neurobehavioral Outcomes. Brain Imaging Behav 4(1): 22-34.

31. Gofton TE, Young GB (2012) Sepsis-associated encephalopathy. Nat Rev Neurol 8(10): 557-566.

32. Burkhart CS, Siegemund M, Steiner L (2010) Cerebral perfusion in sepsis. Crit Care 14(2): 215.

33. Belet N, Belet U, Incesu L, Uysal S, Özinal S, et al. (2004) Hypoxic-ischemic encephalopathy: Correlation of serial MRI and outcome. Pediatr Neurol 31(4): 267-274.

34. Gordon N (1980) Apparent cerebral atrophy in patients on treatment with steroids. Dev Med Child Neurol 22(4): 502-506.

35. Aoi MC, Hu K, Lo M-T, Selim M, Olufsen MS, Novak V (2012) Impaired Cerebral Autoregulation Is Associated with Brain Atrophy and Worse Functional Status in Chronic Ischemic Stroke. PLoS One 7: e46794.

36. Zahr NM, Mayer D, Rohlfing T, Orduna J, Luong R, et al. (2013) A mechanism of rapidly reversible cerebral ventricular enlargement independent of tissue atrophy. Neuropsychopharmacology 38(6): 11211129.

37. Tsao N, Hsu HP, Wu CM, Liu CC, Lei HY (2001) Tumour necrosis factoralpha causes an increase in blood-brain barrier permeability during sepsis. J Med Microbiol 50(9): 812-821.

38. du Moulin GC, Paterson D, Hedley-Whyte J, Broitman SA (1985) E. coli peritonitis and bacteremia cause increased blood-brain barrier permeability. Brain Res 340(2): 261-268.
39. Bartynski WS, Boardman JF, Zeigler ZR, Shadduck RK, Lister J (2006) Posterior reversible encephalopathy syndrome in infection, sepsis, and shock. Am J Neuroradiol 27(10): 2179-2190.

40. Chen XY, Lam WWM, Ho KN, Fan YH, Wong KS (2006) The frequency and determinants of calcification in intracranial arteries in Chinese patients who underwent computed tomography examinations. Cerebrovasc Dis 21(1-2): 91-97.

41. Demer LL, Tintut Y (2008) Vascular calcification: Pathobiology of a multifaceted disease. Circulation 117(22): 2938-2948.

42. Sangiorgi G, Rumberger JA, Severson A, Edwards WD, Gregoire J, Fitzpatrick LA, et al. (1998) Arterial calcification and not lumen stenosis is highly correlated with atherosclerotic plaque burden in humans: A histologic study of 723 coronary artery segments using nondecalcifying methodology. J Am Coll Cardiol 31: 126-133.

43. Mackey RH, Venkitachalam L, Sutton-Tyrrell K (2007) Calcifications, arterial stiffness and atherosclerosis. Adv Cardiol 44: 234-244.

44. Sutter R, Chalela JA, Leigh R, Kaplan PW, Yenokyan G, et al. (2015) Significance of Parenchymal Brain Damage in Patients with Critical Illness. Neurocrit Care 23(2): 243-252.

45. Brazzelli MG, Sandercock P, Wardlaw J (2011) Evidence-based guideline: the role of diffusion and perfusion MRI for the diagnosis of acute ischemic stroke: report of the Therapeutics and Technology Subcommittee of the American Academy of Neurology. Neurology 76: 2036-2037.

46. Prass K, Meisel C, Höflich C, Braun J, Halle E, Wolf T, et al. (2003) Strokeinduced immunodeficiency promotes spontaneous bacterial infections and is mediated by sympathetic activation reversal by poststroke T helper cell type 1-like immunostimulation. J Exp Med 198(5): 725-736.

47. Kraft P, Drechsler C, Schuhmann MK, Gunreben I, Kleinschnitz C (2015) Characterization of Peripheral Immune Cell Subsets in Patients with Acute and Chronic Cerebrovascular Disease: A Case-Control Study. Int J Mol Sci 16(10): 25433-254349.

48. Westendorp WF, Nederkoorn PJ, Vermeij J-D, Dijkgraaf MG, van de Beek D (2011) Post-stroke infection: a systematic review and meta-analysis. BMC Neurol 11: 110.

49. Hilker R, Poetter C, Findeisen N, Sobesky J, Jacobs A, et al. (2003) Nosocomial pneumonia after acute stroke: Implications for neurological intensive care medicine. Stroke 34(4): 975-981.

50. Taccone FS, Su F, Pierrakos C, He X, James S, et al. (2010) Cerebral microcirculation is impaired during sepsis: an experimental study. Crit Care 14(4): R140.

51. Hshieh TT, Dai W, Cavallari M, Guttmann CR, Meier DS, et al. (2016) Cerebral blood flow MRI in the nondemented elderly is not predictive of post-operative delirium but is correlated with cognitive performance. J Cereb Blood Flow Metab 37(4): 1386-1397.

52. Alsop D, Detre J, Golay X, Günther M (2015) Recommended implementation of arterial spin-labeled perfusion MRI for clinical applications: A consensus of the ISMRM perfusion study group and the European consortium for ASL in dementia.. Magn Reson Med 73(1): 102-116.

53. Cho TH, Nighoghossian N, Wiart M, Desestret V, Cakmak S, et al. (2007) USPIO-enhanced MRI of neuroinflammation at the sub-acute stage of ischemic stroke: Preliminary data. Cerebrovasc Dis 24: 544-546. 
Creative Commons Attribution 4.0 International License

For possible submissions Click Here

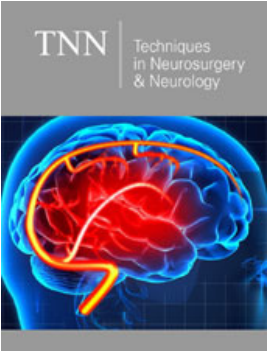

Techniques in Neurosurgery \& Neurology

\section{Benefits of Publishing with us}

- High-level peer review and editorial services

- Freely accessible online immediately upon publication

- Authors retain the copyright to their work

- Licensing it under a Creative Commons license

- Visibility through different online platforms 